\title{
Evolution of Eurasian teleconnection pattern and its relationship to climate anomalies in China
}

\author{
Ning Wang • Yaocun Zhang
}

Received: 25 September 2013/Accepted: 5 May 2014/Published online: 20 May 2014

(C) The Author(s) 2014. This article is published with open access at Springerlink.com

\begin{abstract}
The Eurasian teleconnection pattern (EU) is a major mode of low-frequency variability in the Northern Hemisphere winter, with notable impacts on the temperature and precipitation anomalies in Eurasia region. To investigate the structure, life cycle and dynamical mechanisms of EU pattern, diagnostic analyses are conducted to clarify EU pattern evolution, with an emphasis on EU development and decay. In the developing stage, a geopotential height anomaly over North Atlantic emerges 6 days before EU peak phase and other three geopotential height anomalies appear one by one in the following days. During this period, all geopotential height anomalies experience considerable growth and the four-center structure of EU pattern forms 2 days before peak phase. The obvious wave train structure appears at $300 \mathrm{hPa}$. The EU pattern growth is driven by both relative vorticity advection and transient eddy fluxes. After the peak phase, the geopotential height anomaly over North Atlantic becomes weak as it decays earlier than other anomaly centers, which leads to the classic three-center structure of EU teleconnection pattern. The complete life cycle of EU pattern experience considerable growth and decay within 10 days. During the decaying stage, the horizontal divergence and the transient eddy fluxes play important roles. Additionally, the relationship of EU pattern to winter climate anomalies in China is also analyzed focusing on the decaying stage. The impact of EU pattern on temperature and precipitation in China are significant in 2-4 days after EU peak phase and the distribution of temperature and precipitation anomaly has obvious regional differences.
\end{abstract}

N. Wang · Y. Zhang $(\bowtie)$

School of Atmospheric Sciences, Nanjing University,

Nanjing 210093, China

e-mail: yczhang@nju.edu.cn
Keywords Eurasian teleconnection pattern - Spatial structure $\cdot$ Temporal evolution $\cdot$ Climate anomalies in China

\section{Introduction}

Teleconnection patterns represent the atmospheric lowfrequency variability at regional or global scale. The early studies of low frequency patterns can be traced back to 1930s when Walker and Bliss (1932) identified the North Atlantic and North Pacific Oscillations. Since then a number of prominent teleconnection patterns have been recognized in the Northern Hemisphere $(\mathrm{NH})$ using correlation analysis and orthogonally rotated principal component analysis (Wallace and Gutzler 1981; Esbensen 1984; Mo and Livezey 1986; Barnston and Livezey 1987; Ding and Wang 2005). Many studies suggested that there are five significant teleconnection patterns in $500 \mathrm{hPa}$ geopotential height field in NH winter: the eastern Atlantic pattern (EA), the Pacific/North American pattern (PNA), the western Atlantic pattern (WA), the western Pacific pattern (WP) and the Eurasian pattern (EU) (Wallace and Gutzler 1981; Horel 1981; Barnston and Livezey 1987). Among these five significant patterns, Eurasian teleconncetion pattern (EU) is an important low-frequency pattern with well-known impacts on the atmospheric circulation and climate anomalies in the Eurasia region as the three action centers of EU pattern are located over Eurasia continent.

The horizontal structure of teleconncetion patterns consists of "seesaw" and/or wavelike structures at monthly mean time scale (Nakamura et al. 1987). The Eurasian pattern belongs to the wavelike structure group as the action centers align along particular latitude circle. At monthly time scale, EU pattern emerges as three action 
centers with alternating signs: Scandinavia and Poland, Siberia, and Japan. The present work focuses on the structure and evolution of EU pattern at daily time scales, which can give us a better understanding of the developing and decaying of EU pattern. The dynamical processes that account for the growth and decay of EU pattern will also be examined.

Climate effect of teleconnection patterns is one of the most attractive topics recently. Park et al. (2010, 2011) pointed out that the intensity and frequency of the East Asian cold surges are closely related to the state of AO and Eurasia blocking systems. Sung et al. (2006) reported a possible delayed impact of the winter North Atlantic oscillation (NAO) on the following East Asian summer monsoon precipitation. Several studies revealed that the PNA pattern strongly affects winter temperature and precipitation across the United States (Leathers et al. 1991; Notaro et al. 2006). Meanwhile, the impact of EU pattern on Eurasia climate during $\mathrm{NH}$ winter has also investigated. Some studies suggested that EU pattern contributes to the decadal shift of geopotential height in winter 1989 when the phase of EU pattern reversed from positive to negative (Watanabe and Nitta 1999; Ohhashi and Yamazaki 1999). In addition, EU pattern plays an important role in EAWM variability, with significantly correlation to the Siberia High and temperature anomaly in eastern China (Gong et al. 2001). Positive EU phase is accompanied by a strong northerly wind and a sudden descent of temperature in south China and Korea while the probability distribution of cold/warm events is dependent on the phase of the EU pattern (Sung et al. 2009). Furthermore, Tachibana et al. (2007) found that the snow-accumulation event occurrence tended to coincide with negative EU pattern in Tokyo winter. They suggested that the low surface air temperature in Tokyo associated with negative EU pattern is favorable to snowfall and snow accumulation. Liu and Chen (2012) found that the positive phase of EU pattern is accompanied by an intensified subtropical jet, deepened East Asian trough, lower temperature and less precipitation in East China. While previous studies mainly focus on the spatial distribution of climate anomalies over China at monthly time scale, climate anomalies accompanied with the evolution of EU pattern are still unknown. In this study, we investigate the temporal evolution of temperature and precipitation anomalies during the growth and decay periods of EU pattern. As we will show, the daily analysis unfolds more details of EU climate effects.

The rest of this paper is organized as follows. In Sect. 2, the data used in this study are described, and the EU index and peak phase of EU pattern are defined. The life cycle, temporal evolution, vertical structure and the dynamical features for the positive and negative phases of EU pattern are shown in Sect. 3. The spatial differences and temporal evolutionary feature of temperature and precipitation anomalies in China during positive and negative phases of EU pattern are illustrated in Sect. 4. Conclusions are presented in Sect. 5.

\section{Data and methods}

In this study, we employ the National Centers for Environmental Prediction/National Center for Atmospheric Research (NCEP-NCAR) reanalysis data for cold season (November-March) from 1950 to 2010, including geopotential height field and wind field. The horizontal resolution of the data is $2.5^{\circ} \times 2.5^{\circ}$ in longitude and latitude. The daily field was used after removing the climatological annual cycle which is defined by a 31-day running mean of the 60-year mean value for each calendar day (Sung et al. 2009). Daily averaged temperature and precipitation data from 756 observation stations in China are also used. The data covers the period from 1 January 1950 to 31 July 2010.

The daily EU index is calculated using the method defined by Wallace and Gutzler (1981):

$$
\begin{aligned}
E U(W G)= & -\frac{1}{4} Z^{*}\left(55^{\circ} \mathrm{N}, 20^{\circ} \mathrm{E}\right)+\frac{1}{2} Z^{*}\left(55^{\circ} \mathrm{N}, 75^{\circ} \mathrm{E}\right) \\
& -\frac{1}{4} Z^{*}\left(40^{\circ} \mathrm{N}, 145^{\circ} \mathrm{E}\right)
\end{aligned}
$$

where $Z^{*}$ represents the normalized $500 \mathrm{hPa}$ geopotential height anomaly. The positive phase appears as the EU index exceeds zero, while the negative phase appears when EU index is below zero. The peak phase of EU pattern is defined as the day when daily EU index is a local maximum and exceeds one standard deviation (Sung et al. 2009), indicating that the geopotential height anomalies of the EU action centers attain maximal value over Eurasia continent. If two or more peak phases occur in less than 15 days, only the first peak is counted to ensure the independence of each peak phase.

\section{General features of $\mathrm{EU}$ pattern}

\subsection{Life cycle}

To show the life cycle of EU pattern, we examine the composites of EU index around the positive and negative peak phase at daily time scale. These composites consist of totally 272 positive phases and 285 negative phases. As shown in Fig. 1, for both phases, the amplitude of EU pattern grows rapidly from lag -5 days and attains maximum in lag 0 days, then decays. The decaying period takes more days than growing period. The whole life cycle of EU pattern experiences about 10 days. The composites of positive and negative EU index show opposite structure. 


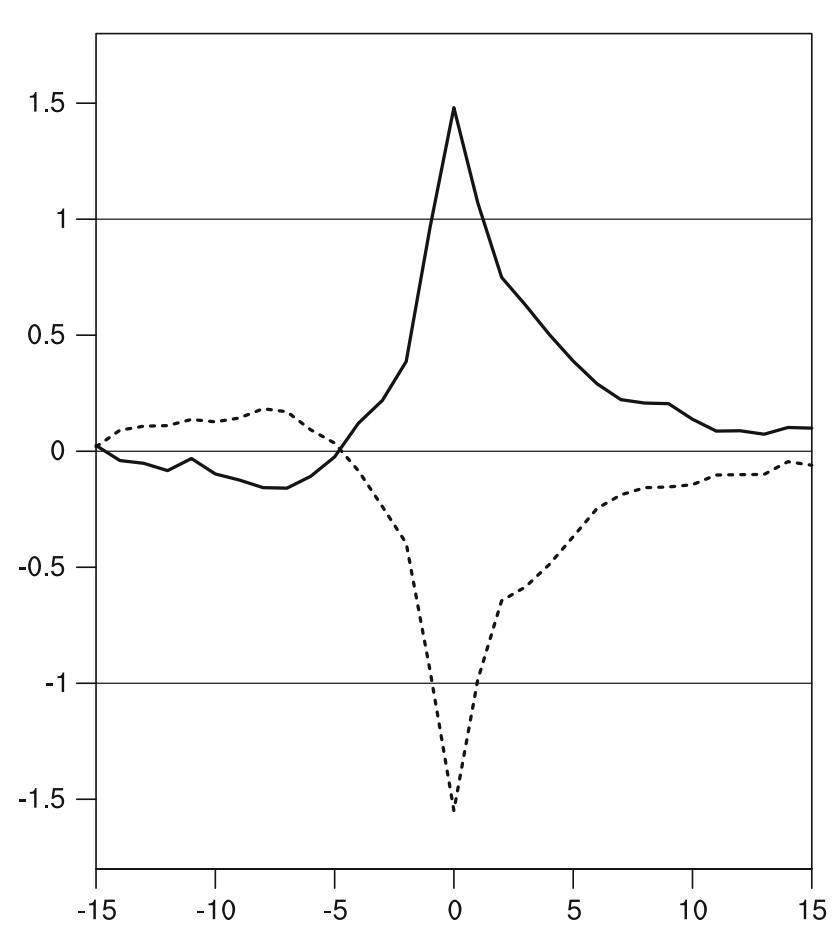

Fig. 1 Composite of EU index from lag -15 to 15 days. Lag 0 day denotes the peak phase. The solid line denotes the positive phase and dashed line is the negative phase

We further calculate the daily composites of $500 \mathrm{hPa}$ geopotential height anomalies for positive EU phase extending from lag -6 to lag +6 days (Fig. 2). At lag -6 days, a geopotential height anomaly of EU pattern emerges over North Atlantic in positive phases. During lag -5 to lag -2 days, this positive geopotential height anomaly center gradually intensifies while other three centers with one positive anomaly over Japan and two negative anomalies over Poland and Siberia emerge gradually. The complete four-center structure of EU pattern is formed at lag -2 days when the geopotential height anomaly over North Atlantic attains maximum. During the following 2 days, there is a rapid decline in the strength of the anomaly over North Atlantic, whereas other anomaly centers of EU pattern experience considerable growth. Except for the center over North Atlantic, other three anomaly centers attain maximum by lag 0 days, corresponding to the EU pattern peak phase. The decay of EU pattern commences after lag 0 days. The anomaly center over North Atlantic becomes weak as it decays 2 days earlier than other centers, resulting in the absence of this center at monthly time scale. By lag +2 days, the strength of the anomalies over Poland, Siberia and Japan decay rapidly, and the anomaly over North Atlantic almost disappears at the same time, which leads to the classic three-center structure of EU pattern over Eurasia in the decaying stage. After lag +2 days, the geopotential height anomalies over Eurasia decay substantially one after another and the EU pattern disappears after lag +5 days.

In the upstream region of EU pattern, a weak positive anomaly appears over the east coast of North America. This anomaly shows no change during the whole life cycle of the EU pattern. Another interesting feature is seen over north Tibetan Plateau where a weak negative geopotential height anomaly appears at lag -6 days. With the growth of EU pattern, the negative anomaly propagates eastward without growth. At lag -2 days, this anomaly merges with that over Japan, and the anomaly reinforces afterwards. After the EU peak phase, the negative anomaly continues to propagate eastward until it reaches Pacific Ocean and maintains there for a few days.

We also examine the composites of negative phases for the $500 \mathrm{hPa}$ geopotential height anomalies (Fig. 3). It is evident that both phases of EU pattern have similar temporal evolution feature. The negative phases start with a negative geopotential height anomaly located over North Atlantic and the structure is opposite to the positive phases.

The geopotential height anomaly fields illustrated in Figs. 2 and 3 show the feature of EU pattern growth and decay in positive and negative phases. The action centers of EU pattern develop one after another from lag -6 days and the overall structure of EU pattern completes before EU pattern gets maximum amplitude. EU pattern exhibits four significant anomaly centers in developing period over North Atlantic, Poland, Siberia and Japan, which are quite different from previous studies. The anomaly center over North Atlantic is weaker and decays 2 days earlier than other centers. For this reason, this center almost disappears after the peak phase when the classic three-center structure is obvious. Since the locations of all anomalies are fixed during EU pattern lifetime, the phase velocity of the wave train approximates to zero which is consistent with the conclusion that the EU pattern is a quasi-stationary wave (Hoskins and Karoly 1981).

\subsection{Vertical structure}

Previous studies suggested that the EU pattern has an equivalent barotropic structure in vertical direction (Wallace and Gutzler 1981; Ohhashi and Yamazaki 1999; Liu and Chen 2012). These results were obtained by comparing low and high level circulation anomaly fields. In this study, we directly show the vertical cross sections of geopotential height field and potential temperature along the anomaly centers with respect to positive EU phase (Fig. 4). Negative phase is opposite to that of positive phase (figure not shown). Throughout the troposphere, there are alternating warm-ridge and cool-trough. The important feature seen in Fig. 4 is that all the maximum anomaly centers appear at $300 \mathrm{hPa}$. 

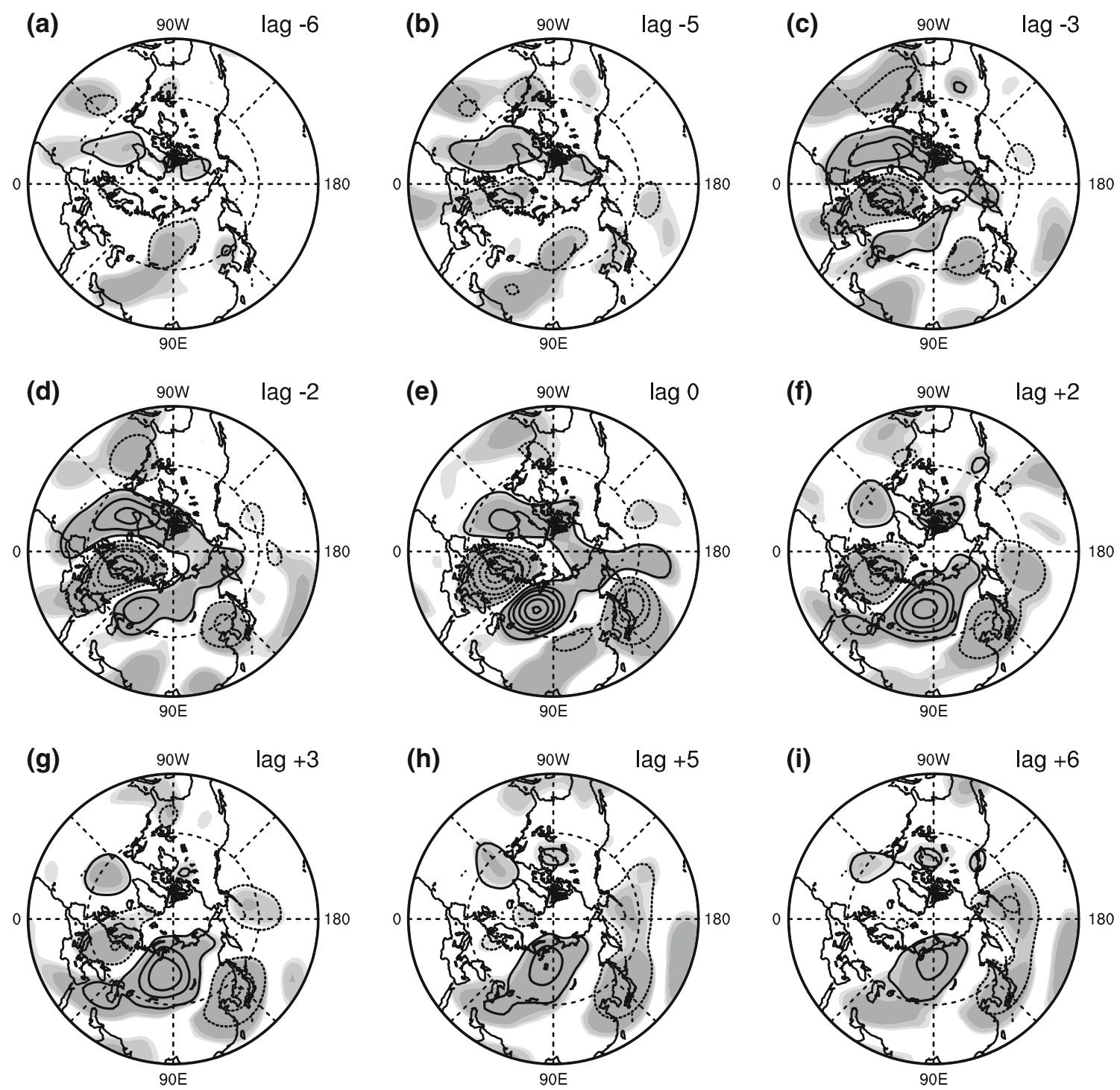

Fig. 2 The $500 \mathrm{hPa}$ geopotential height anomaly field for positive EU phase at lag -6 days (a), lag -5 days (b), lag -3 days (c), lag -2 days (d), lag 0 days (e), lag +2 days (f), lag +3 days (g), lag +5 days $(\mathbf{h})$ and lag +6 days (i). Solid contours are positive and

In lag -6 days (Fig. 4a), a weak positive anomaly emerges over North Atlantic. During the following 3 days, the positive anomaly strengthens and new anomalies form downstream farther over Eurasia continent. Geopotential height anomalies almost have barotropic structure but the nascent geopotential height anomalies tilt a little eastward and potential temperature anomalies tilt westward in lower troposphere which shows a little baroclinic structure. In lag -2 days, the four-center structure of EU pattern is completed while the anomaly center over North Atlantic attains maximum. During the following 2 days (Fig. 4e), the anomalies over Eurasia experience considerable reinforcement, and all the previously existing geopotential height anomalies become barotropic. The anomaly centers dashed contours are negative. The contour interval is $30 \mathrm{~m}$. Light and heavy shadings indicate significant variation above 95 and $99 \%$ confidence level, respectively

of EU pattern attain their maximum except for that over North Atlantic. The geopetential anomalies over Eurasia get maximal value at lag 0 days. An obvious decay of EU pattern commences after that.

The former analyses indicate that the baroclinicity may play a role in the beginning of the geopotential height anomaly, but the maintenance and development of the anomalies are mainly ascribed to barotropic instability. Barotropic instability grows by extracting kinetic energy from horizontal wind shear in a jet-like current (Holton and Hakim 2012). Over Eurasia continent, the EU pattern aligns along the climatologic position of polar front jet, which can explain the presence of maximum amplitude of EU pattern over Eurasia continent. 

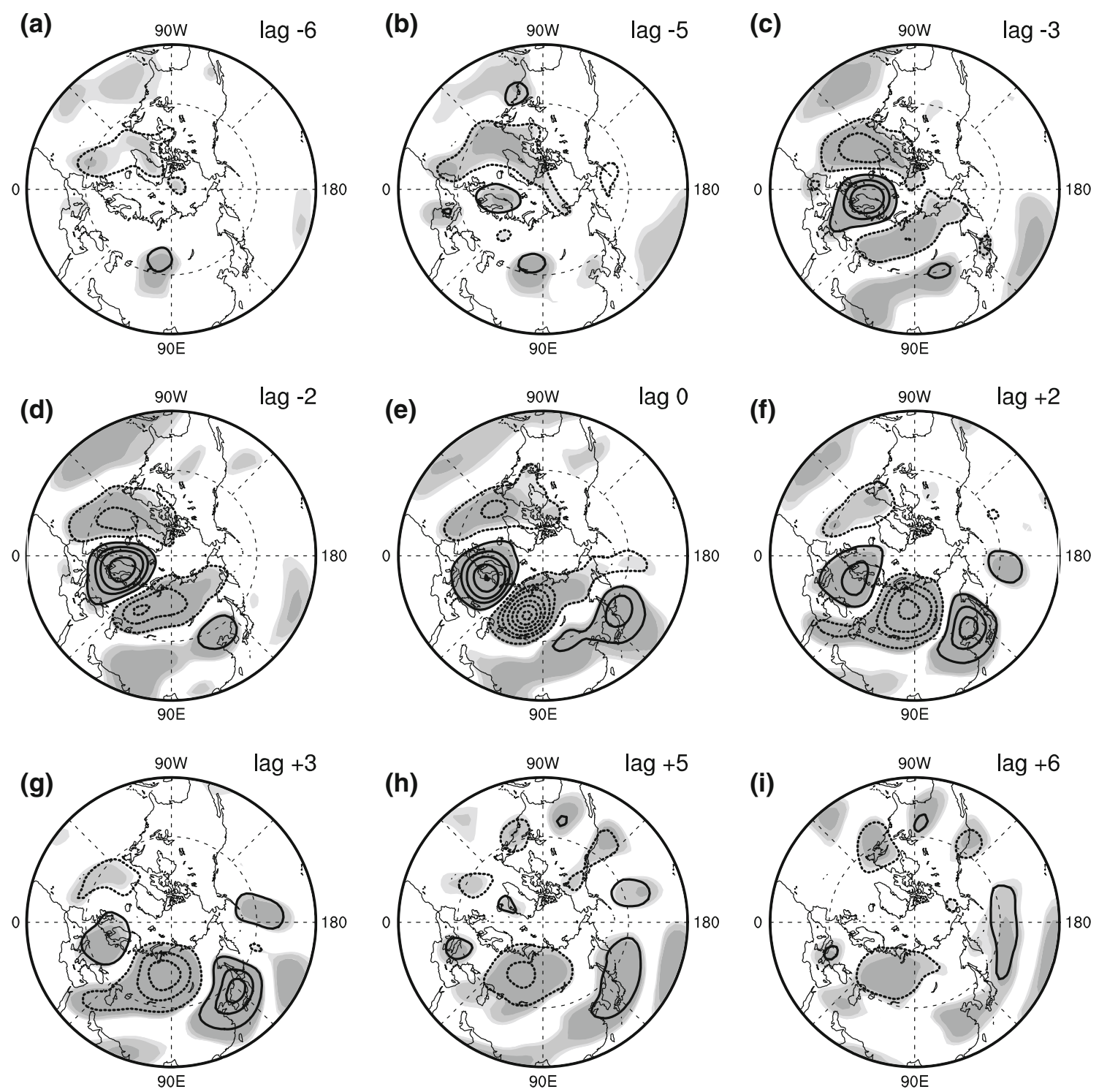

Fig. 3 The same as Fig. 2, but for negative EU phase

\subsection{The dynamics of EU pattern growth and decay}

To investigate the dynamical features of the EU pattern, we calculate each term in stream-function tendency equation (Cai and van den Dool 1994; Feldstein 2002, 2003; Feldstein and Dayan 2008; Franzke and Feldstein 2005). This equation can be written as:

$\frac{\partial \psi}{\partial \mathrm{t}}=\sum_{i=1}^{6} \xi_{i}+R$

where $\Psi$ is the $500 \mathrm{hPa}$ stream-function, and residual term $\mathrm{R}$ is small, indicating that Eq. (1) is reasonably well balanced, and $\xi_{i}(\mathrm{i}=1,2, \ldots, 6)$ are defined as follows:

$$
\begin{aligned}
\xi_{1}= & \nabla^{-2}\left(-\frac{\mathbf{v}}{a} \frac{d f}{d \theta}\right) \\
\xi_{2}= & \nabla^{-2}(-[\overline{\mathbf{v}}] \cdot \nabla \zeta-\mathbf{v} \cdot \nabla[\bar{\zeta}]) \\
\xi_{3}= & \nabla^{-2}\left(-\overline{\mathbf{v}^{*}} \cdot \nabla \zeta-\mathbf{v} \cdot \nabla \overline{\zeta^{*}}\right) \\
\xi_{4}= & \nabla^{-2}(-(\mathrm{f}+\bar{\zeta}) \nabla \cdot \mathbf{v}-\zeta \nabla \cdot \overline{\mathbf{v}}) \\
\xi_{5}= & \nabla^{-2}\left(-\mathbf{v}_{r} \cdot \nabla \zeta\right)+\nabla^{-2}\left\{-\nabla \cdot\left(\mathbf{v}_{d} \zeta\right)\right\} \\
\xi_{6}= & \nabla^{-2}\left\{-\mathbf{k} \cdot \nabla \times(\omega \partial \overline{\mathbf{v}} / \partial p\}+\nabla^{-2}\{-\mathbf{k} \cdot \nabla \times(\bar{\omega} \partial \mathbf{v} / \partial p)\}\right. \\
& +\nabla^{-2}\{-\mathbf{k} \cdot \nabla \times(\omega \partial \overline{\mathbf{v}} / \partial p)\}+\nabla^{-2}\{-\mathbf{k} \cdot \nabla \times(\bar{\omega} \partial \mathbf{v} / \partial p)\} \\
& +\nabla^{-2}\left\{-\mathbf{k} \cdot \nabla \times\left(\omega^{\prime} \partial \mathbf{v}^{\prime} / \partial p\right)\right\}
\end{aligned}
$$

where $\zeta$ is the relative vorticity, $\mathbf{v}$ the horizontal wind vector, $\mathrm{v}$ the meridional wind component, $\omega$ the vertical 

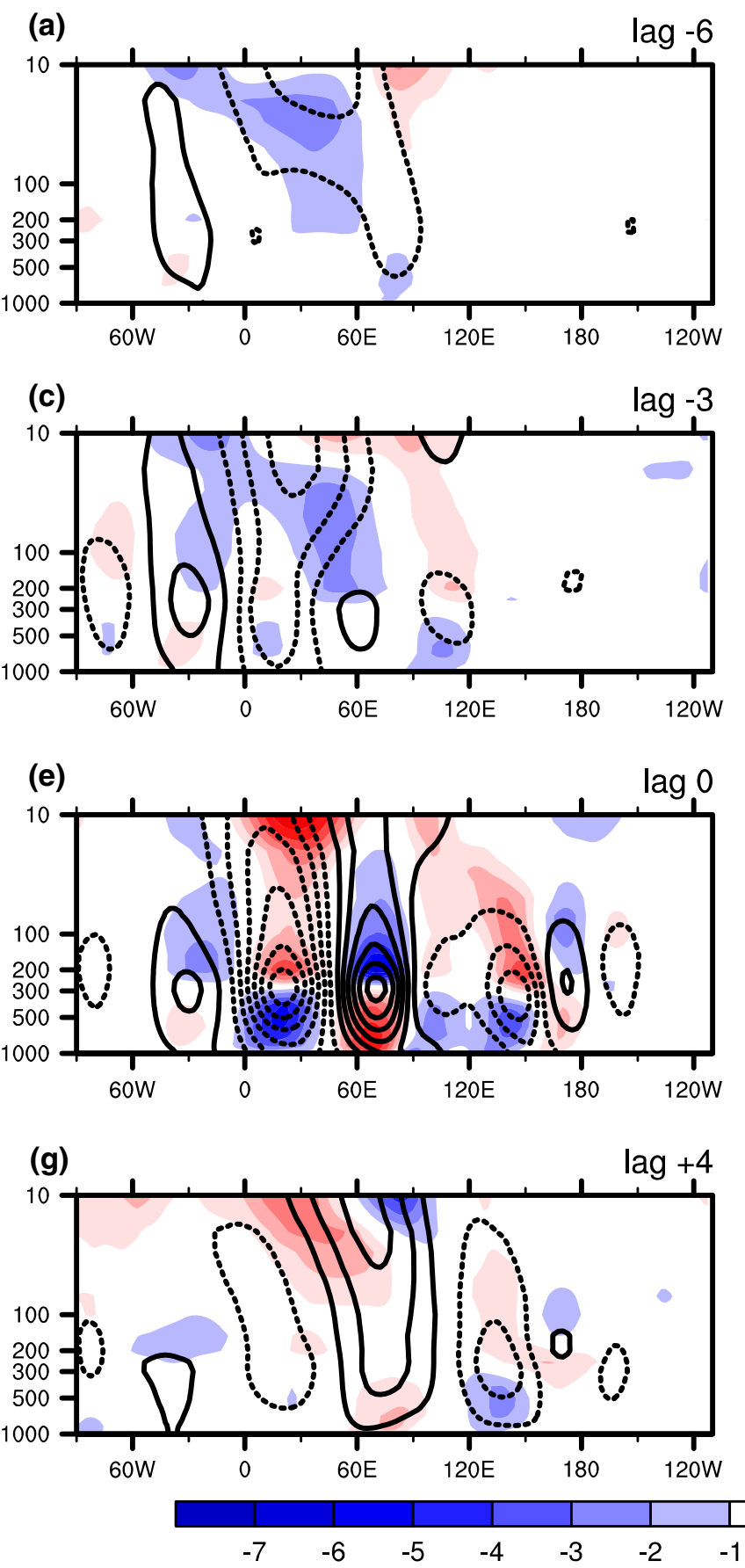

Fig. 4 Vertical cross sections of geopotential height anomaly (contours) and potential temperature anomaly (shadings) along the center of each anomaly for positive EU phase at lag -6 days (a), lag -5 days (b), lag -3 days (c), lag -2 days (d), lag 0 days (e), lag

wind component, $a$ the Earth's radius, $f$ the Coriolis parameter, and $\theta$ is the latitude. The subscripts ' $r$ ' and ' $d$ ' denote the rotational and divergent components of the horizontal wind, respectively. An overbar defines the timemean, and deviations from the time-mean are indicated by a prime. The square bracket denotes the zonal average, the asterisk a deviations from the zonal average. On the right-

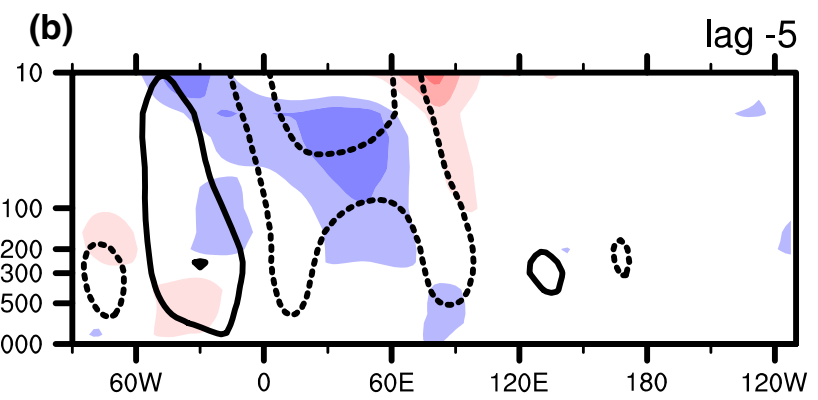

(d)

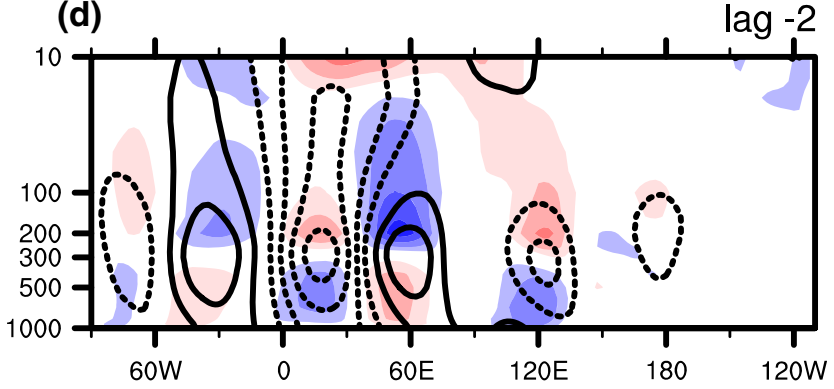

(f)

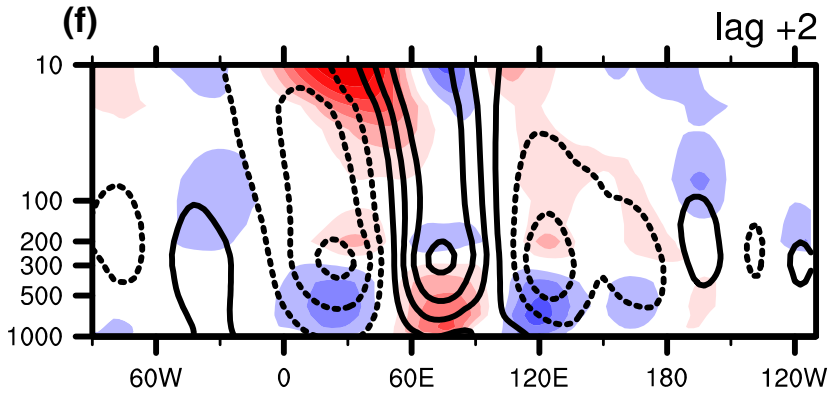

(h)

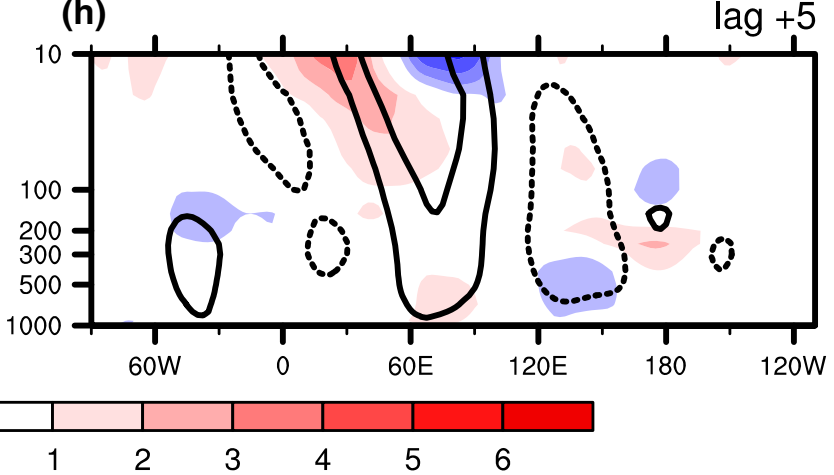

+2 days (f), lag +4 days (g) and lag +5 days (h). The contour interval is $30 \mathrm{~m}$, solid contours are positive, dashed contours are negative

hand side (RHS), $\xi_{1}$ is planetary vorticity advection by the anomalies, $\xi_{2}\left(\xi_{3}\right)$ the relative vorticity advection which involves the interaction between the anomalies and the zonally symmetric (asymmetric) climatological flow, $\xi_{4}$ the divergence term, and $\xi_{5}$ the forcing term by transient eddy vorticity flux. The quantity $\xi_{6}$ is the forcing due to the tilting term, which is not discussed because it is small 
Fig. 5 Projections of each terms on rhs of stream-function tendency equation onto streamfunction anomaly field at lag 0 days for the positive EU phase: a rhs of stream-function euqation, $\sum_{i=1}^{5} \xi_{i}$ (rsh, solid line), and $\partial \psi / \partial t$ (lhs, dashed line); $\mathbf{b} \xi_{1}$ (term1, solid line) and $\xi_{2}$ (term2, dashed line); $\mathbf{c} \xi_{3}$ (term3, solid line) and $\xi_{4}$ (term4, dashed line); $\mathbf{d} \xi_{5}$ (term5, solid line). The ordinate has been multiplied by $5 \times 10^{6} \mathrm{~s}^{-1}$ (a)

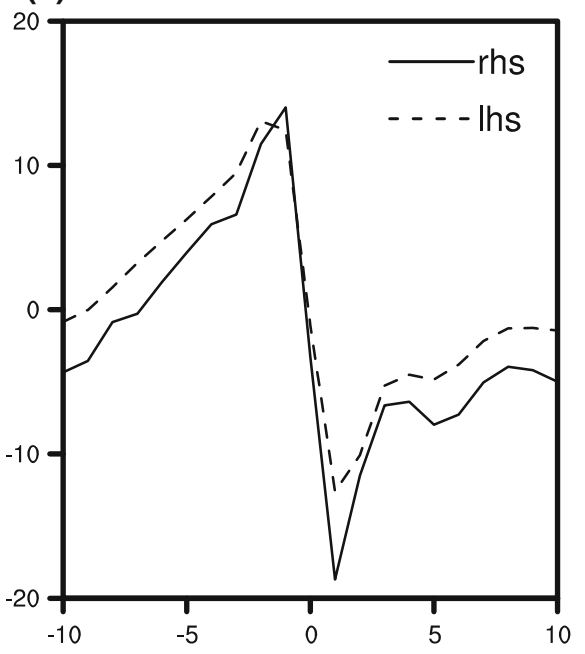

(c)

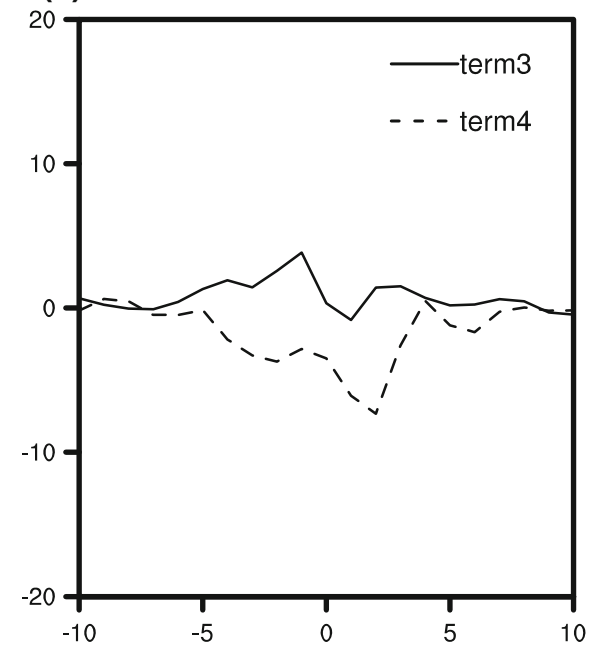

(b)

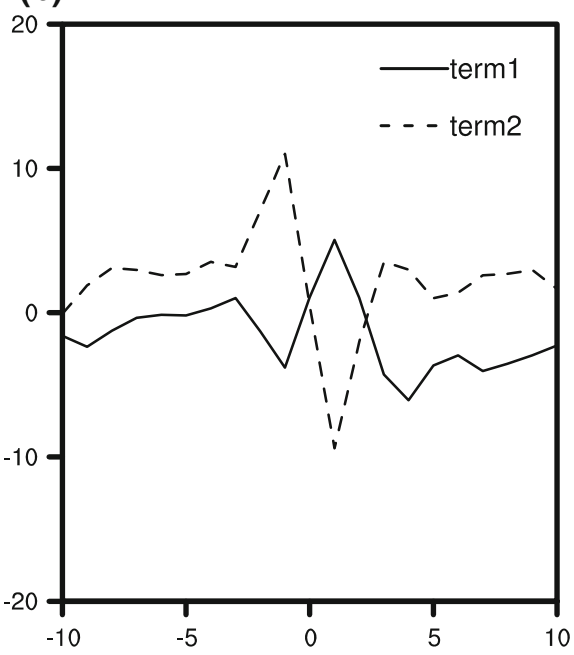

(d)

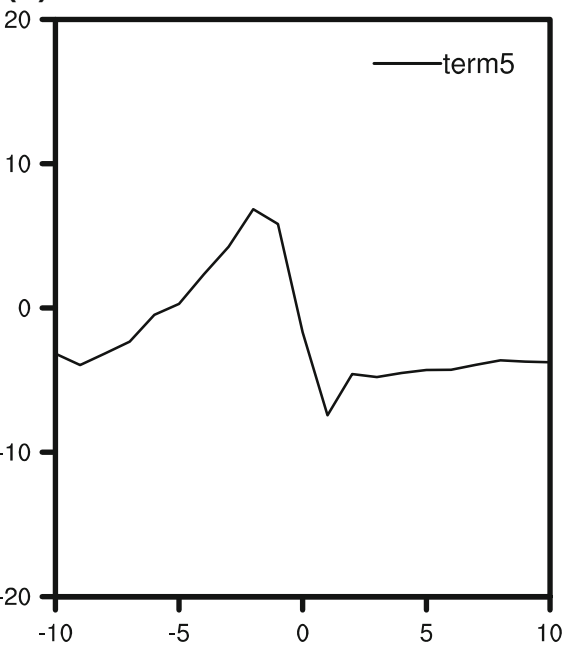

(Feldstein and Dayan 2008). More detailed information about this equation can be seen in Feldstein and Dayan (2008).

The composite analysis of each term in the streamfunction tendency equation can help us to understand which processes contribute to the growth, maintenance and decay of EU pattern. We project each term on the RHS of Eq. (1) onto the anomalous stream-function pattern at lag 0 days (Fig. 5), using the same procedure as Feldstein and Dayan (2008). As discussed by Feldstein and Dayan (2008), this projection represents the influence of the individual term on the time rate of change of streamfunction. It also represents the change of geopotential height field as the stream-function field is directly proportional to geopotential height field on the geostrophic approximation. We have examined the stream-function anomaly field during the life cycle of EU pattern which shows almost the same structure as geopotential height anomaly field. Since the projection for both phases of EU are found to be similar, only the results for the positive EU phase are presented.

We first compare the projection of the sum of five terms on the RHS with the projection of the term on the left-hand side (LHS) of the stream-function tendency equation (1) (Fig. 5a). As the tilting term and the residual term $\mathrm{R}$ on the RHS are excluded, we should make sure the equation is well balanced. As can be seen in Fig. 5a, there is only a little difference between the RHS and LHS through the growth and decay period of EU pattern. The difference between the projection of RHS and LHS reflects the influence of the excluded terms. Therefore, at most days the projection of RHS is close to LHS especially near lag 0 days, which means the five terms can well explain the stream function tendency. The contribution of the tilting term and residual $\mathrm{R}$ in the projection is small in most lag days.

Figure $5 \mathrm{~b}-\mathrm{d}$ shows the projection of five terms on the RHS. As can be seen, the planetary vorticity advection 
term (term 1) contributes a lot to the maintenance and decay of EU pattern, but contributes a little to the EU pattern growth. The relative vorticity advection involving the interaction between the anomalies and the zonally symmetric/asymmetric climatological flow terms (term 2/term 3) both contribute to the growth of EU pattern, especially term 2 . But the relative vorticity advection contributes very little to the decay of EU pattern. The decay of EU results primarily from horizontal divergence (term 4) and nonlinear transient eddy fluxes (term 5). Meanwhile the nonlinear transient eddy flux (Fig. 5d) contributes substantially to the growth of EU pattern. This feature is different from other teleconnection patterns such as PNA and NAO. PNA is driven by linear terms and NAO is dominated by nonlinear terms in Eq. (1) (Feldstein 2002, 2003) while the EU pattern growth is primarily driven by linear processes and nonlinear processes together. In addition, the transient eddy flux plays an important role in both the growth and decay of EU pattern.

\section{Surface climate anomalies in China associated with EU pattern}

In the former analyses, we examined the life cycle, temporal evolution features and dynamics of EU teleconnection pattern. In this section, we explore the association of EU pattern with surface climate anomalies in China. We use composite analysis to investigate the surface air temperature and precipitation anomaly fields, followed by the temporal variation of regional averaged temperature and precipitation anomaly with respect to positive and negative phases of EU pattern in NH winter. To better capture the strong impact of EU pattern on the distribution of surface temperature and precipitation anomalies, we choose the days when EU index remains above one standard deviation for at least 5 days for composite analysis (Horel 1985; Mo 1986; Feldstein 2002, 2003; Feldstein and Dayan 2008; Walter and Graf 2006).

The composite patterns of temperature and precipitation anomaly over China for both positive and negative EU phases are shown in Fig. 6. The temperature anomaly shows uniform variation all over China, while precipitation anomaly shows east-west difference for both phases. A perceptible decrease of temperature occurs during the positive events, with three evident centers located in North China, Northeast China and South China. The precipitation anomaly field is characterized by a remarkable decrease in East China and considerable increase in Southwest China for positive EU phase. A reverse of the temperature and precipitation anomaly occurs during negative events. Over all, positive EU phase is associated with a cold-dry condition in East China, while a warm-wet condition occurs in negative phase.
In order to examine the temporal variation of the precipitation and temperature anomaly corresponding to developing and decaying stage of EU pattern, we calculate the regional averaged temperature and precipitation anomalies for composite analysis. For the temperature field, we choose three regions: North China, Northeast China and South China; while for precipitation field, we choose two regions: East China and Southeast China. All the stations which we use to average are above $95 \%$ confidence level and these stations are shown in Fig. 6 with circles of different colors.

The temporal variation feature of regional averaged temperature anomaly in North China, Northeast China and South China are shown in Fig. 7. In the case of positive phase, a reduction of temperature emerges near lag -3 days when EU pattern undergoes significant growth. In the negative composites, there is a significant increase of temperature in the same period. The temperature variation is almost reversed between positive and negative EU phase according to the opposite geopotential height anomalies between positive and negative EU patterns. The variations of regional averaged temperature anomaly show similar tendencies in three regions, however there are still remarkable differences. During the positive phase, the maximum temperature fall appears in lag +2 days in North China (Fig. 7a), lag +3 days in Northeast China (Fig. 7b) and lag +4 days in South China (Fig. 7c), respectively. The maximum temperature rise occurs at the same time during the negative events. The extent of temperature fall/rise associated with EU pattern is smaller in south region (South China) than north region (North China and Northeast China). This result is supported by the findings in Sung et al. (2009). The influence of EU pattern is caused directly by the low-frequency anomaly (Sung et al. 2009) and the geopotential height anomalies associated with EU pattern align along middle-high latitude, which makes the temperature variation in north region larger than in south region.

Figure 8 shows the temporal variation feature of precipitation anomaly in East China and Southwest China as the EU pattern evolves. The precipitation anomaly exhibits more fluctuant feature compared to temperature anomaly. In Southwest China, precipitation is enhanced apparently during the positive phase (Fig. 8b), while a reduction of rainfall happens in East China (Fig. 8a). Similar to the variation feature of temperature anomaly, the precipitation anomaly are nearly reversed between positive and negative phases of EU pattern. The maximum of precipitation anomaly emerges in lag $+2 / \mathrm{lag}+3$ days in Southwest China and in lag +4 days in East China, respectively. The extent of precipitation increase/decrease is larger in East China than Southwest China.

As mentioned above, the variation of EU pattern leads the variation of surface temperature and precipitation over 

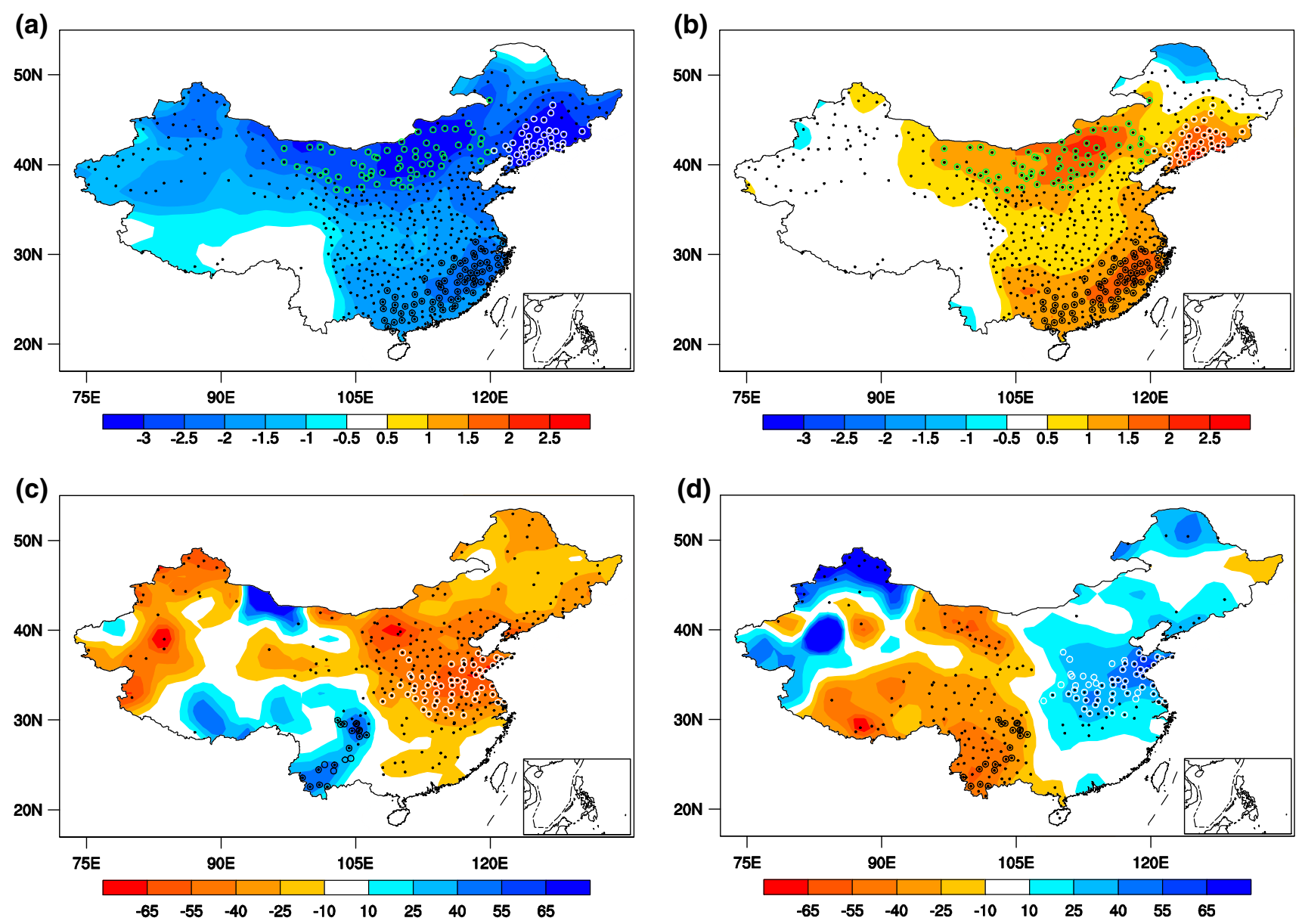

Fig. 6 Temperature anomaly (a, b) and percentage changes of precipitation $(\mathbf{c}, \mathbf{d})$ in China for positive $(\mathbf{a}, \mathbf{c})$ and negative $(\mathbf{b}, \mathbf{d}) \mathrm{EU}$ phase. Filled circles represent the stations significant at $95 \%$ level. Empty circles are the stations which are averaged to show the time evolutionary feature in Figs. 7 and 8. Three centers in temperature

China a few days. In addition, the lead-time has remarkable regional difference. It is possible to take daily EU index as a precursor for surface temperature and precipitation anomalies over China.

\section{Conclusions}

This study focuses on the structure, temporal evolution feature and dynamical features of EU pattern and its influence on winter temperature and precipitation anomalies over China at daily time scale.

In the classical definition, the EU teleconnection pattern is characterized by a three action centers over Eurasia continent. The daily composite analysis of EU pattern reveals more other features. Different from previous studies, EU pattern exhibits four significant action centers in developing stage but three centers in decaying stage. The formation of EU action center begins 6 days before EU

field (a, b): North China (green circles), Northeast China (white circles), and South China (black circles). Two centers in precipitation field (c, d): East China (white circles) and Southwest China (black circles)

gets maximum amplitude. From lag -6 to lag -2 days, the geopotential height anomalies of EU pattern emerge one after another. Two days before the peak phase, the structure of EU pattern is formed and its four significant geopotential height anomalies are located over North Atlantic, Poland, Siberia and Japan. The center located over North Atlantic is absent at monthly time scale. The possible reason is that this center is weaker and decays earlier than other centers. In the decaying stage of EU pattern, the anomaly center over North Atlantic almost disappears and then the classic three-center structure is obvious. In the whole lifetime of EU pattern evolution, the positions of the action centers change very little, indicating that the EU pattern is a quasistationary wave. The negative phase shows similar temporal evolution feature with positive phase except for reversing the geopotential height anomalies.

The vertical structure of EU pattern shows equivalent barotropic feature. However, in the beginning period of anomalies, there is a little eastward tilt in geopotential 
Fig. 7 Temperature anomalies averaged in North China (a), Northeast China (b) and South China (c) around the peak phase of EU pattern from lag -5 to lag +7 days. Solid line is positive phase and dashed line is negative phase. The unit is ${ }^{\circ} \mathrm{C}$. The solid circles indicate significant variation above $95 \%$ confidence level

Fig. 8 Precipitation anomalies averaged in East China (a) and Southwest China (b) around the peak phase of EU pattern from lag -5 to lag +7 days. Solid line is positive $\mathrm{EU}$ phase and dashed line is negative EU phase. The unit is $0.1 \mathrm{~mm}$. The solid circles indicate significant variation above $95 \%$ confidence level (a)

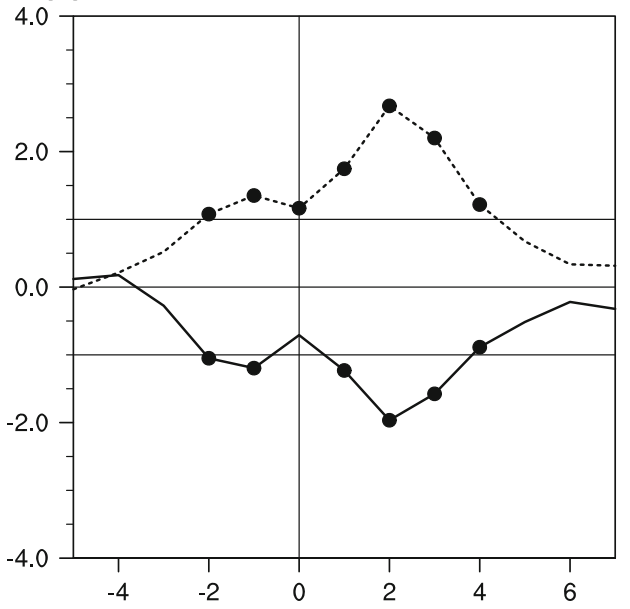

(c)

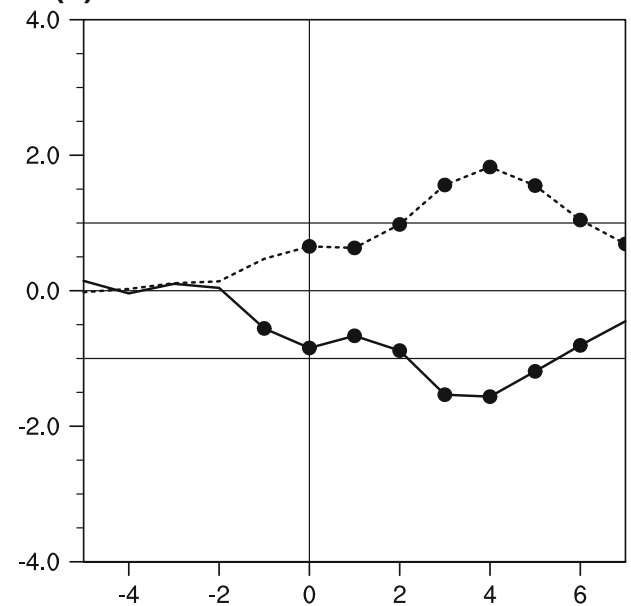

(a)

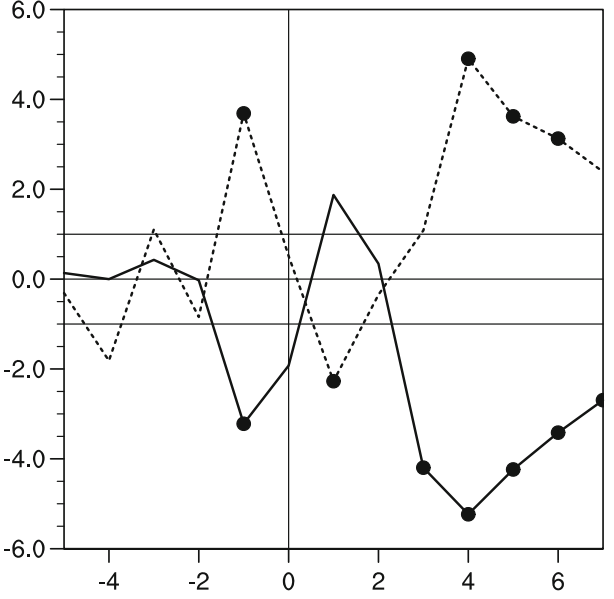

(b)

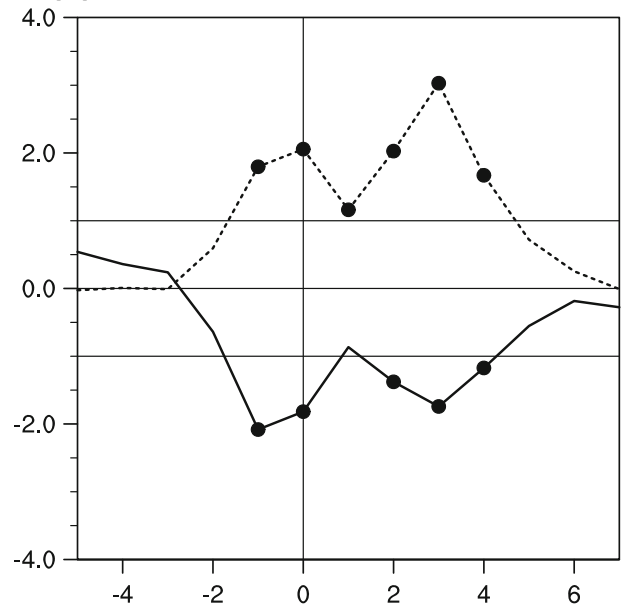

(b)

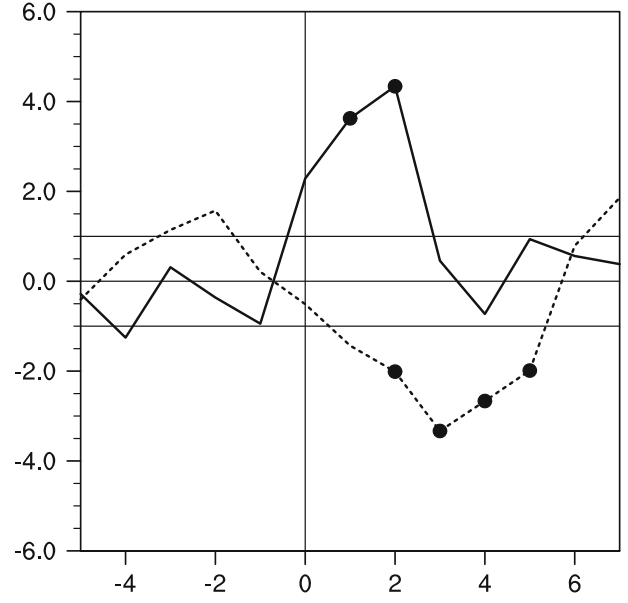

height field and westward tilt in potential temperature field. These baroclinic structure anomalies become barotropic structure in the following days with considerable reinforcement. This feature indicates that the baroclinic instability may influence the anomalies when they have just formed while the barotropic instability plays an important role in the developing and maintenance stages of the anomalies of EU pattern. 
We examine the dynamical features that account for the growth and decay of EU pattern by investigating the temporal evolution of five terms on the right-hand side of stream-function tendency equation during the life cycle of EU pattern. The analysis indicates that EU pattern is driven by linear advection terms and nonlinear transient eddy vorticity flux together. The decay of EU is caused primarily by horizontal divergence and transient eddy vorticity flux. It is noticeable that in the whole life cycle of EU pattern, the transient eddy vorticity flux plays an important role.

With the strengthening of geopotential height anomalies over Eurasia continent, EU pattern exerts great influence on surface climate anomalies over broad region. Significant temperature fall is apparent all over China during the positive phase with three strong temperature fall regions in North China, Northeast China and South China. In negative EU phase, there is significant temperature rise. The variation of regional averaged temperature anomaly shows similar tendencies in the mentioned three regions. With the growth of EU pattern, temperature fall/rise commences 3 days before positive/negative EU peak phase. The extent of temperature anomaly in South China is smaller compared to North China and Northeast China. This difference is mainly because that EU pattern aligns along the middle-high latitude region. In addition, the largest temperature fall/rise appears in 2, 3 and 4 days in North China, Northeast China and South China, respectively.

The distribution of precipitation anomaly shows eastwest differences. East China has less/more rain than usual in positive/negative EU phase, while Southwest China is opposite. The variation of regional averaged precipitation anomaly is more fluctuant than temperature anomaly. The largest precipitation anomaly appears in 2-3 and 4 days after the peak phase of EU pattern in East China and Southwest China, respectively. The extent of precipitation rise/fall is larger in East China compared to Southwest China. As EU pattern varies earlier than the temperature and precipitation anomaly over East Asia, it is quite possible to take daily EU index as a precursor for surface temperature and precipitation anomalies over China.

\footnotetext{
Acknowledgements The authors acknowledge the editor and anonymous reviewers who provided valuable suggestions for improving our manuscript. This work was jointly supported by the National Basic Research Program of China (973 Program, grant 2012CB955901), the National Natural Science Foundation of China (grant 41130963), and the Jiangsu Collaborative Innovation Center for Climate Change.
}

Open Access This article is distributed under the terms of the Creative Commons Attribution License which permits any use, distribution, and reproduction in any medium, provided the original author(s) and the source are credited.

\section{References}

Barnston AG, Livezey RE (1987) Classification, seasonality and persistence of low-frequency atmospheric circulation patterns. Mon Weather Rev 115(6):1083-1126

Cai M, Van Den Dool HM (1994) Dynamical decomposition of low frequency tendencies. J Atmos Sci 51:2086-2100

Ding Q, Wang B (2005) Circumglobal teleconnection in the Northern Hemisphere Summer. J Clim 18(17):3483-3505

Esbensen SK (1984) A comparison of intermonthly and interannual teleconnections in the $700 \mathrm{mb}$ geopotential height field during the Northern Hemisphere winter. Mon Weather Rev 112(10):2016-2032

Feldstein SB (2002) Fundamental mechanisms of PNA teleconnection pattern growth and decay. Q J R Meteorol Soc 128(4430):4440

Feldstein SB (2003) The dynamics of NAO teleconnection pattern growth and decay. Q J R Meteorol Soc 129:091-924

Feldstein SB, Dayan U (2008) Circumglobal teleconnections and wave packets associated with Israeli winter precipitation. Q J R Meteorol Soc 134(631):455-467

Franzke C, Feldstein SB (2005) The continuum and dynamics of Northern Hemisphere teleconnection patterns. J Atmos Sci 62:3250-3267

Gong DY, Wang SW, Zhu JH (2001) East Asian winter monsoon and Arctic oscillation. Geophys Res Lett 28:2073-2076

Holton JR, Hakim GJ (2012) An introduction to dynamic meteorology. Academic Press, London, pp 229-230

Horel JD (1981) A rotated principal component analysis of the interannual variability of the Northern Hemisphere $500 \mathrm{mb}$ height field. Mon Weather Rev 109(10):2080-2092

Horel JD (1985) Persistence of the $500 \mathrm{mb}$ height field during Northern Hemisphere winter. Mon Weather Rev 113(11):2030-2042

Hoskins BJ, Karoly DJ (1981) The steady linear response of a spherical atmosphere to thermal and orographic forcing. J Atmos Sci 38:1179-1196

Leathers DJ, Yarnal B, Palecki MA (1991) The Pacific/North American teleconnection pattern and United States climate. Part I: Regional temperature and precipitation associations. J Clim 4:517-528

Liu YY, Chen W (2012) Variability of the Eurasian teleconnection pattern in the Northern Hemisphere winter and its influences on the climate in China. Chin J Atmos Sci 36(2):423-432

Mo KC (1986) Quasi-stationary states in the Southern Hemisphere. Mon Weather Rev 114(5):808-823

Mo KC, Livezey RE (1986) Tropical-extratropical geopotential height teleconnections during the Northern Hemisphere winter. Mon Weather Rev 114(12):2488-2515

Nakamura H, Tanaka M, Wallace JM (1987) Horizontal structure and energetics of Northern Hemisphere wintertime teleconnection patterns. J Atmos Sci 44(22):3377-3391

Notaro M, Wang WC, Gong W (2006) Model and observational analysis of the northeast US regional climate and its relationship to the PNA and NAO patterns during early winter. Mon Weather Rev 134(11):3479-3505

Ohhashi Y, Yamazaki K (1999) Variability of the Eurasian pattern and its interpretation by wave activity flux. J Meteorol Soc Jpn $77: 495-511$

Park TW, Ho CH, Yang S, Jeong JH (2010) Influences of Arctic oscillation and Madden-Julian oscillation on cold surges and heavy snowfalls over Korea: a case study for the winter of 2009-2010. J Geophys Res 115:D23122. doi:10.1029/ 2010JD014794

Park TW, Ho CH, Yang S (2011) Relationship between the Arctic oscillation and cold surges over East Asia. J Clim 24:68-83 
Sung MK, Kwon WT, Baek HJ, Boo KO, Lim GH, Kug JS (2006) A possible impact of the North Atlantic oscillation on the East Asian summer monsoon precipitation. Geophys Res Lett 33:L21713. doi:10.1029/2006GL027253

Sung MK, Lim GH, Kwon WT (2009) Short-term variation of Eurasian pattern and its relation to winter weather over East Asia. Int J Climatol 29(5):771-775

Tachibana Y, Nakamura T, Tazou N (2007) Interannual variation in snow-accumulation events in Tokyo and its relationship to the Eurasian pattern. Sci Online Lett Atmos 3:129-131

Walker GT, Bliss EW (1932) World weather. V Mem R Meteorol Soc 4:53-84
Wallace JM, Gutzler DS (1981) Teleconnections in the geopotential height field during the Northern Hemisphere winter. Mon Weather Rev 109(2):784-812

Walter K, Graf HF (2006) Life cycles of North Atlantic teleconnections under strong and weak polar vortex conditions. Q J R Meteorol Soc 132(615):467-483

Watanabe M, Nitta T (1999) Decadal changes in the atmospheric circulation and associated surface climate variations in the Northern Hemisphere winter. J Clim 12(2):494-510 